\title{
Entropic Lattice Boltzmann Method on Non-uniform Grids
}

\author{
C. Shyam Sunder and V. Babu \\ Department of Mechanical Engineering, \\ Indian Institute of Technology, \\ Madras, India 600036. \\ vbabu@iitm.ac.in
}

\begin{abstract}
The entropic lattice Boltzmann method (ELBM) has recently been shown to be capable of simulating incompressible flows with good accuracy and enhanced stability. However, the method requires that uniform grids be used, which makes it prohibitively expensive for simulating large Reynolds number flows. In this paper, an interpolation scheme is proposed to extend the applicability of this method to arbitrary non-uniform meshes, while retaining the salient features of ELBM such as stability and computational efficiency. The new scheme is used to simulate flow in a lid driven cavity as well as flow past a square cylinder and is shown to largely reduce the grid requirements. The results of the simulation agree very well with other numerical experimental data available in the literature
\end{abstract}

Keywords: Entropic Lattice Boltzmann ISLB.

\section{Introduction}

In the last decade the Lattice Boltzmann method (LBM) has attracted a lot of attention and is fast emerging as an alternative to finite volume and finite element techniques. Lattice Boltzmann methods have been successful in simulating many simple and complex hydrodynamics including turbulent flows[1]. The main attractions of LBM are ease of implementation, high computational efficiency and easy parallelizability. There are many variations of LBM in use, among which are finite volume Lattice Boltzmann method, multiple relaxation Lattice Boltzmann methods, interpolation supplemented Lattice Boltzmann method, and entropic Lattice Boltzmann method.

The Lattice Boltzmann Equation (LBE) initially originated as a floating point recast of the evolution equation of lattice-gas cellular automaton dynamics. The simplest form of LBE, namely, the Lattice Bhatnagar Gross Krook (LBGK) form, can be written as follows.

$$
f_{i}\left(x+c_{i} \delta_{i}, t+\delta_{t}\right)-f_{i}(x, t)=-\frac{1}{\tau}\left\{f_{i}(x, t)-f_{i}^{e q}(x, t)\right\}
$$


where

$$
c_{i}= \begin{cases}0, & i=0, \\ c\{\cos ((i-1) \pi / 2), \sin ((i-1) \pi / 2)\}, & i=1,2,3,4 \\ \sqrt{2} c\{\cos [(i-5) \pi / 2+\pi / 4], \sin ((i-5) \pi / 2+\pi / 4)\}, & i=5,6,7,8\end{cases}
$$

where $c=\partial x / \partial t$ is the lattice speed, $f_{i}(x, t)$ represents the probability for a particle moving in the direction $c_{i}$ to reside at the location $x$, at time $t$ and $f_{i}^{e q}$ is the equilibrium distribution corresponding to the particular velocity and density. The right hand side of the equation represents the single relaxation time model collision process with $\tau$ representing the dimensionless relaxation time. The local hydrodynamic quantities are given by

$$
\rho=\sum f_{i}, \quad \rho u=\sum f_{i} c_{i}
$$

One of the shortcomings of the LBM is numerical instability. The reason for the instability is that no bounds are imposed on the values $f_{i}^{e q}$ and $f_{i}$ during the collision process making it possible for $f_{i}$ to take negative values depriving it of any physical sense [2]. One of the ways to ensure the positivity of $f_{i}$ is to define the corresponding equilibrium value as a minimum of a convex function, known as the $\mathrm{H}$ function, under the constraint of the local conservation laws [2].

Apart from the stability issue, another well known problem associated with the discrete velocity models is non-adherence to the equation of the state [3],[4]. In these models, the local equilibrium entropy does not obey the thermodynamic definition of the temperature being a function of entropy and energy [4]. These issues were addressed in the formulation of the ELBM [4],[5],[6],[7]. This is discussed in the next section.

As described by Eq. 1, particles at a lattice site undergo collision followed by advection. The left hand side of Eq. 1 can be split into two parts viz. calculation of $f_{i}\left(x, t+\delta_{t}\right)$ (updating post collision values) and calculation of $f_{i}\left(x+c_{i} \delta_{t}, t+\delta_{t}\right)$ (advection in the direction $c_{i}$ ). This advection however can be done only to the neighboring lattice sites at a distance of $c_{i} \delta_{t}$ which constrains the lattice used for the simulation to be a uniform square mesh. This issue was addressed by He et al [8] for a 9 velocity LBE model, wherein the extension of LBE for nonuniform meshes was outlined. The objective of the present work is to extend the ELBM method to non-uniform meshes also using the methodology outline by He et al [8].

\section{Entropic Lattice Boltzmann Method}

The construction of the entropic Lattice Boltzmann method (ELBM) is based on a good choice of the discrete velocities, the $H$ function and an expression for the equilibrium values[9]. The discrete form of the $H$ function is derived from the continuous Boltzmann $H$ function given by $\int F \ln F d c$ where $F(\boldsymbol{x}, c)$ is the single particle distribution function, $\boldsymbol{x}$ is the position vector and $c$ is the continuous 
velocity. For $2 \mathrm{D}$ athermal cases, the discrete form the $H$ function can be written as

$$
H_{\left(W_{i}, f_{i}\right)}=\sum_{i=0}^{8} f_{i} \ln \left(\frac{f_{i}}{W_{i}}\right)
$$

where $f_{i}$ represents discrete velocities and $W_{i}$ the weights associated with each direction. The weights in one dimension are $\{1 / 6,2 / 3,1 / 6\}$ for the directions 'Right', 'Zero' and 'Left' respectively and the weights for higher dimensions can be constructed by multiplying the weights associated with each component direction[10]. The equilibrium value of the discrete velocity is the minimizer of the corresponding $H$ function under the constraints of local conservation laws given by Eq. 3. The explicit solution for the $f_{i}^{e q}$ in $D$ dimensions is

$$
f_{i}^{e q}=\rho W_{i} \prod_{j=1}^{D}\left(2 u_{j}-\left(\sqrt{1+3 u_{j}^{2}}\right)\right)\left(\frac{2 u_{j}+\sqrt{1+3 u_{j}^{2}}}{1-u_{j}}\right)^{c_{i j} / c_{s}}
$$

where $j$ is the index for spatial directions, $c_{s}$ is the velocity of sound and the exponent $c_{i j} / c_{s}$ can only take the values $+1,0,-1$. $H$ can be guaranteed to remain non-increasing everywhere by a two step process explained in Fig. 1. The population is changed first by keeping the $H$ constant. In the second step dissipation is introduced to decrease $H$. It can be guessed that in the steady state i.e. when $f_{i}^{e q}=f_{i}$, value of $H$ remains constant. The BGK form of the collision in ELBM is

$$
f_{i}\left(x+c_{i} \delta_{t}, t+\delta_{t}\right)-f_{i}(x, t)=\alpha \beta\left[f_{i}(x, t)-f_{i}^{e q}(x, t)\right]
$$

where $\beta=6 /(1+6 \nu)$ is the relaxation frequency with $\nu$ being the kinematic viscosity. The parameter $\alpha$ can be calculated by solving the following nonlinear equation

$$
H(f)=H(f+\alpha \Delta)
$$

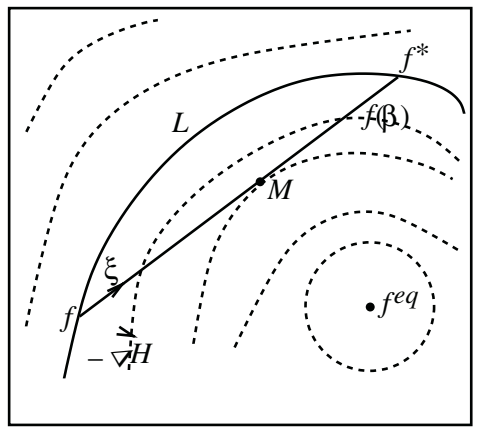

Fig. 1. Graphical representation of the collision process. The curves shown represent lines of constant entropy and $\xi$ represents the collision integral. The first step of the collision process is the calculation of the point $f^{*}$, by solving equation Eq. 7 . Then $f(\beta)$ is determined using 'over-relaxation' based on the value of $\beta[9]$. In case of BGK collision, the point $\mathrm{M}$ coincides with $f^{e q}$ 
where $\Delta$ represents the bare departure from the local equilibrium i.e. $\Delta=f^{e q}-f$. The value of $\alpha$ is usually close to 2 (and equal to 2 when $f=f^{e q}$ ). The procedure for explicit calculation of $\alpha$ was described by Ansumali and Karlin [10]. By using different value for $\alpha$ at each lattice site, the relaxation time is adjusted locally to ensure compliance with the $H$ theorem. This guarantees the positivity of the distribution function, which gives the scheme good non-linear stability.

\section{Entropic Lattice Boltzmann Method on Non-uniform Grids}

The ELBM as outlined above is restricted to uniform square meshes. With increasing Reynolds number, this results in a tremendous increase in the total number of points required and hence an increase in computation time. Moreover, ELBM is computationally more expensive than LBM because of multiple calculations of ' $\ln ^{\prime}$ function at every lattice point. The present work aims at extending the applicability of ELBM to arbitrary nonuniform meshes which can help reduce the total number of grid points required for a simulation.

The distribution functions $(f)$ in LB models are continuous functions in both space and time, although they are used to represent population of particles locally. This gives us scope for interpolation schemes to be used in the interior of the computational domain and extrapolation schemes at the boundary.

In short, the implementation of ELBM on nonuniform grids can be explained in the following steps:

1. Start with an initially guessed flow field.

2. For each lattice site, calculate $f^{e q}$ according to Eq. 5 and hence find $\Delta$ $\left(=f^{e q}-f\right)$.

3. Calculate $\alpha$ by solving the nonlinear equation 7 .

4. Perform collision according to equation 6 .

5. Advect the post collision values to appropriate neighboring nodes (imaginary square lattice on which the LB automaton resides).

6. Calculate the values on the 'actual' lattice sites (mesh used in the simulation) by means of quadratic interpolation.

7. Apply the necessary boundary conditions.

8. Goto step 2.

It should be noted that step 5 can be combined with step 6 , eliminating the need to store and maintain the values of $f_{i}$ on the nodes of the imaginary lattice. Computationally ELBM can be divided into three kernels viz. collision (steps 2, 3,4 ), advection and interpolation (steps 5 and 6 ), boundary conditions (step 7). In general, collision accounts for about $90 \%$ of the total computational load, and advection and interpolation about $8 \%$.

In the case of ELBM on square grids i.e. without interpolation being done on the grid, the time spent in collision accounts for almost $98 \%$ of the computational load. This shows that the overhead associated with the introduction 
of the interpolation routine is not very high. It will be shown in the coming sections that the savings incurred in terms of grids is enormous and more than compensates for the extra calculation being done in interpolation.

\section{Enhancement in Reynolds Number}

Another advantage of using nonuniform or rectangular meshes is the possibility of increasing the Reynolds number of the simulation for a given number of grid points and without any loss of stability. The Reynolds number of the simulation, Re is equal to $U L_{\text {char }} / \nu$ where $U$ is the reference velocity, $\nu$ is kinematic viscosity in lattice units and $L_{\text {char }}$ is the characteristic length for the flow in grid units and is equal to $N_{\text {char }} \delta^{\text {mesh }} / \delta^{\text {automaton }}$. Here $\delta^{m e s h}$ is the spacing of the computational grid and $\delta^{\text {automaton }}$ is the spacing of the grid on which the automaton resides. $N_{\text {char }}$ is the number of grid points per characteristic length measured on the latter grid. Now for square uniform meshes, $\delta^{\text {automaton }}=\delta^{\text {mesh }}$. However for rectangular and nonuniform meshes, $\delta^{\text {mesh }}$ can be chosen to be greater than $\delta^{\text {automaton }}$ resulting in increase in the Reynolds number of the simulation by a factor of $\delta^{\text {mesh }} / \delta^{\text {automaton }}$. The use of nonuniform and rectangular grids does not affect the stability of the system (or the LB automaton) as the $\delta^{\text {automaton }}$ is not being altered. Hence for a given number of grid points, the Reynolds number is enhanced. Moreover, by choosing different levels of grid spacing it is possible to greatly reduce the number grid points required for the simulation.

\section{Results and Discussion}

In this section, results from numerical simulations using ELBM on non-uniform grids are presented. Both steady (lid driven cavity flow) and unsteady flows (flow past a square) are simulated to demonstrate the suitability of the methodology.

\subsection{Lid Driven Cavity Flow}

In this classical test problem, fluid is contained within three stationary walls while the fourth (top) wall moves with a certain velocity. This results in the formation of a large primary vortex near the center of the cavity and smaller vortices (two or three, depending on the Reynolds numbers) near the corners. A non-uniform mesh with about 60-100 grid points along each side of the cavity has been used for the simulations. Diffusive boundary condition [11] has been used for all the four walls. In the diffusive boundary condition, the corners can be associated with either of the walls or the normal can be made to be inclined at a $45^{\circ}$ angle. In the current implementation, the corners are treated as points belonging to the side walls (left or right). The other choice for the association of corner points is also possible and would not affect the flow by a great extent as three of the four diagonal speeds $(f)$ do not propagate into the domain. The results of the simulation compare well with those available in the literature as shown in Table 1. 
Table 1. Comparison of results for thelid driven cavity problem

\begin{tabular}{|c|c|c|c|c|}
\hline Re & Reference & $\begin{array}{c}\text { Primary vortex } \\
\left(\Psi_{\max }, X, Y\right)\end{array}$ & $\begin{array}{c}\text { Lower left vortex } \\
\left(\Psi_{\max } \times 10^{-5}, X, Y\right)\end{array}$ & $\begin{array}{c}\text { Lower right vortex } \\
\left(\Psi_{\max } \times 10^{-4}, X, Y\right)\end{array}$ \\
\hline 400 & {$[13]$} & $(0.1121,0.5608,0.607)$ & $(1.30,0.0549,0.0510)$ & $(6.19,0.8902,0.1255)$ \\
& {$[14]$} & $(0.1130,0.5571,0.607)$ & $(1.42,0.0508,0.0469)$ & $(6.42,0.8906,0.1250)$ \\
& current & $(0.1120,0.548,0.596)$ & $(1.25,0.04,0.036)$ & $(5.92,0.88,0.112)$ \\
\hline 1000 & {$[13]$} & $(0.1178,0.5333,0.564)$ & $(22.2,0.0902,0.0784)$ & $(16.9,0.8667,0.1137)$ \\
& {$[14]$} & $(0.1179,0.5313,0.562)$ & $(23.1,0.0859,0.0781)$ & $(17.5,0.8594,0.1094)$ \\
& current & $(0.1175,0.536,0.564)$ & $(18.2,0.052,0.040)$ & $(17.1,0.852,0.108)$ \\
\hline 5000 & {$[13]$} & $(0.1214,0.5176,0.537)$ & $(135,0.0784,0.1373)$ & $(30.3,0.8075,0.0745)$ \\
& {$[15]$} & $(0.1190,0.5117,0.535)$ & $(136,0.0703,0.1367)$ & $(30.8,0.8086,0.0742)$ \\
& current & $(0.095,0.508,0.528)$ & $(132,0.066,0.128)$ & $(30.2,0.8025,0.068)$ \\
\hline
\end{tabular}

\subsection{Flow Past a Square}

Here, flow past a square immersed in a stream of fluid is considered. As shown in the figure, a square of side $L_{\text {char }}$ is placed symmetrically at a location $10 L_{\text {char }}$ down stream of the inlet $A B$. The size of the computational domain is $45 L_{\text {char }}$ in the direction of the flow and $25 L_{\text {char }}$ perpendicular to the flow (resulting in a blockage ratio of $4 \%$ ). The side CD represents the outlet boundary, and

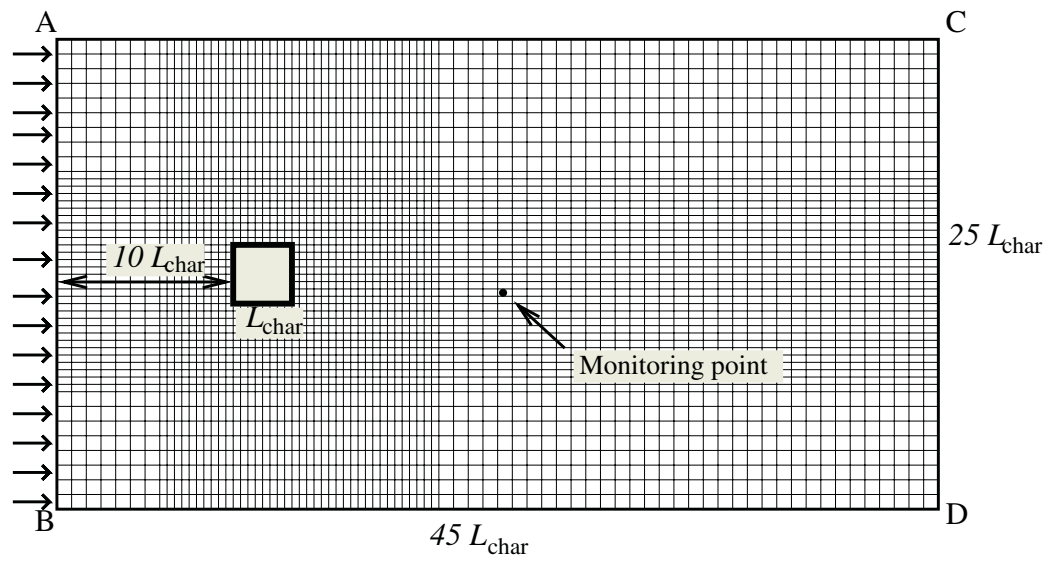

Fig. 2. Flow past a Square

the sides $B C$ and $D A$ are free stream boundaries. This flow becomes unsteady for Reynolds numbers above 40 or so. If the dominant frequency of the vortex shedding is denoted by $n_{f}$ then the Strouhal number of the flow is $n_{f} L_{\text {char }} / U_{\infty}$, where $U_{\infty}$ is the free stream velocity. In the past, several techniques such as the Finite Difference, Finite Volume and Lattice Boltzmann methods, have successfully predicted the Strouhal number - Reynolds number relation at low and 
moderate Reynolds numbers [17],[18]. At high Reynolds numbers, conventional techniques face difficulties such as enormous grid requirements, lack of stability and lack of a good exit boundary condition [1]. The difficulty with the exit boundary condition can be handled by increasing the domain size in the direction of flow, albeit, at a tremendous increase in the computational cost. In contrast, the non-linear stability of the entropic Lattice Boltzmann method together with lesser grid requirements gives an added advantage over the normal LB methods. Thus, the usage of non-uniform grids with ELBM makes it possible to simulate the flow at high Reynolds numbers and with a reduced computational cost. Table 2 shows the variation of Strouhal number with Reynolds number as predicted by experiments and numerical calculations. It is evident that the present calculations are able to predict the Strouhal number very well and the agreement is better than that of the results available in the literature so far. The grid sizes used in the present case are much smaller than the grid requirements of normal LBM. At high Reynolds numbers, the $N_{\text {char }}$ used in the current case ranges from $30-50$, whereas the $N_{\text {char }}$ required for normal LBM would be around 128-256 [19] resulting in more than an order of magnitude savings in the grid requirement (Table 2).

Table 2. Comparison of Strouhal number and grid sizes for different Reynolds numbers

\begin{tabular}{|c|c|c|c|c|c|c|}
\hline Re & \multicolumn{3}{|c|}{ Strouhal Number } & \multicolumn{2}{c|}{ Grid Size } \\
\hline & Expt[16] & Current & FV [17] & FD [18] & Current & LBM [19] \\
\hline 250 & $0.140-0.145$ & 0.136 & 0.17 & 0.165 & 60000 & 112500 \\
400 & $0.127-0.136$ & 0.132 & 0.145 & 0.161 & $"$ & $"$ \\
500 & $0.126-0.133$ & 0.132 & 0.174 & - & 240000 & 288000 \\
800 & $0.121-0.126$ & 0.129 & - & - & $"$ & $"$ \\
1000 & $0.120-0.122$ & 0.130 & - & 0.142 & $4.5-5.4 \times 10^{5}$ & $18-73 \times 10^{6}$ \\
2000 & $0.120-0.129$ & 0.131 & - & 0.142 & $"$ & $"$ \\
3000 & $0.123-0.130$ & 0.133 & - & 0.145 & $"$ & $"$ \\
\hline
\end{tabular}

\section{Conclusion}

An interpolation scheme to enable the use of non-uniform meshes is proposed for the entropic lattice Boltzmann method. The method has been successfully used for simulating the steady flow in a lid driven cavity and the unsteady flow over a rectangular cylinder for Reynolds number up to 3000. For the former problem, the location as well as the value of the streamfunction at the center of the vortices is predicted very well by the present calculations, when compared with data available in the literature. For the latter problem, the unsteady vortex shedding frequency predicted by the method compares well with the experimental values reported by Okajima [16]. The proposed interpolation scheme preserves all the salient features of ELBM, like stability, and locality of the collision operator. 
Moreover, the interpolation itself is a very small addition to the overall computational cost. Considerable savings in grid sizes are shown to be possible by comparing with earlier implementations of LBM. This interpolation scheme is quite general and can be easily implemented on different types of grids.

\section{References}

1. S. Succi, The Lattice Boltzmannn Equation for Fluid Dynamics and Beyond, Oxford university press, Oxford, 2001.

2. S. Succi, I. V. Karlin, H. Chen, Colloquium: Role of the $\mathrm{H}$ theorem in lattice Boltzmann hydrodynamic simulations, Rev. Modern Physics 74 (2002).

3. M. Ernst, Discrete Models of Fluid Dynamics, edited by Alves A. S. (World Scientific, Singapore) 1991.

4. S. Ansumali, I. V. Karlin, H. C. Ottinger, Minimal entropic kinetic models for hydrodynamics, Europhys. Lett. 63 (6) (2003) 798-804.

5. B. M. Boghosian, P. J. Love, P. V. Coveney, Iliya V. Karlin, Sauro Succi and Jeffrey Yepez, Galilean-invariant lattice-Boltzmann models with $\mathrm{H}$ theorem, Phys. Rev. E 68, (2003) 025103.

6. B. M. Boghosian, J. Yepez, P. V. Coveney, A. Wagner, Entropic lattice Boltzmann method, Royal Society of London Proceedings Series A, vol. 457, Issue 2007, p.717

7. H. Chen, C. Teixeira, H-theorem and origins of instability in thermal lattice Boltzmann models, Computer Phy. Comm., vol. 129, Issue 1-3, pp.21-31

8. X. He, L. S. Luo, M. Dembo, Some progress in Lattice Boltzmann method :Part 1. Nonuniform Mesh Grids, J. Comp. Phys. 129 (1996) 357-363.

9. I. V. Karlin, A. Ferrante, H. C. Ottinger, Perfect entropy functions of the Lattice Boltzmann method, Europhys. Lett. 47 (2) (1999) 182-188.

10. S. Ansumali, I V. Karlin, Single relaxation time model for entropic lattice Boltzmann methods, Phys. Rev. E 65 (2002) 056312.

11. S. Ansumali, I. V. Karlin, Kinetic boundary conditions in the lattice Boltzmann method, Phys. Rev E 66 (2002) 026311.

12. X. He, L. S. Luo, M. Dembo, Some Progress in Lattice Boltzmann method: Enhancement of Reynolds number in simulation, Physica A 239 (1997) 276-285.

13. S. Hou, Q. Zou, S. Chen, G.D. Doolen, A.C. Cogley, Simulation of Cavity Flow by the Lattice Boltzmann Method, J. Comp. Phys. 118 (1995) 329.

14. R. Schreiber, H.B. Keller, Driven cavity flows by efficient numerical techniques, J. Comp. Phy 49 (1983) 310.

15. U. Ghia, K.N. Ghia, C.Y. Shin, High-Re solutions for incompressible flow using the N.S. equations and a multigrid method, J. Comp. Phys. 48 (1982) 387.

16. A. Okajima, Strouhal numbers of retangular cylinders, J. Fluid Mech. 123 (1982) 379-398.

17. A. Sohankar, C. Norberg, L. Davidson, Simulation of three-dimensional flow around a square cylinder at moderate Reynolds numbers, Phys. Fluids 11 (1999) 288.

18. R. W. Davis, E. F. Moore, Vortex shedding process behind two dimensional buff bodies, J. Fluid Mech. 116 (1982) 475.

19. G. Baskar, V. Babu, Simulation of the Flow Around Rectangular Cylinders Using the ISLB Method, AIAA-2004-2651 (2004). 